\title{
Spreadsheet Add-in for Heat Exchanger Logarithmic Mean Temperature Difference Correction Factors
}

\author{
C.O.C. Oko \\ Dept. of Mechanical Engng \\ University of Port Harcourt \\ PMB 5323, Port Harcourt \\ Rivers State, Nigeria.
}

\author{
E.O. Diemuodeke \\ Dept. of Mechanical Engng \\ University of Port Harcourt \\ PMB 5323, Port Harcourt \\ Rivers State, Nigeria.
}

\author{
M.B. Katsina \\ Dept. of Mechanical Engng \\ University of Port Harcourt \\ PMB 5323, Port Harcourt \\ Rivers State, Nigeria.
}

\begin{abstract}
This paper presents an MS Excel spreadsheet add-in for heat exchanger logarithmic mean temperature difference (LMTD) correction factors for ten heat exchanger configurations. It is a computer tool for determining the LMTD correction factors for complex heat exchanger flow arrangements. The charted correction factors for various flow arrangements were curvefitted using the MS Excel Solver tool. Lagrange interpolation scheme was used to formulate the relevant interpolation formulas for the various flow arrangements considered. The interpolation scheme was programmed in MS Excel Visual Basic for Application as an add-in. Results obtained agree with the values from the correction-factor charts presented in the literature. The add-in is a veritable tool for spreadsheet heat exchanger design and performance analysis for design engineers as well as for educational purposes.
\end{abstract}

\section{General Terms}

Spreadsheet add-in

\section{Keywords}

heat exchangers, LMTD correction factors, curve fitting, spreadsheet add-in

\section{INTRODUCTION}

The process of heat exchange between two fluids that are at different temperatures and separated by a solid wall occurs in many engineering applications. The device used to implement this exchange is called a recuperative heat exchanger. Specific applications may be found in space heating, cooling and airconditioning, power generation, waste heat recovery, separation processes and other chemical processes. The optimal design or selection of heat exchangers is an essential task of the heat exchanger engineer, especially with increasing demand for effective and efficient heat exchangers deployed in engineering systems. This task becomes more involved when complex heat exchangers are to be used. Two of the crucial steps in the thermal design of heat exchangers are the determination of the logarithmic mean temperature difference (LMTD) and the overall heat transfer coefficient (the Uvalue), which are required to compute the required heat transfer surface area $[1,2]$.

The traditional procedure for determining the corrected LMTD is as follows: obtain the inlet and exit temperatures of the hot and cold fluid streams; compute the dimensionless temperature $(P)$ and the ratio of water equivalents of the two streams $(R)$; determine the correction factor $(\Psi)$ from the heat exchanger correction factor charts, which correlates $P, R$ and $\Psi$ as illustrated in Figure 1; compute the counter-flow LMTD; and compute the product of $\Psi$ and LMTD to obtain the corrected LMTD (CLMTD) for the given complex heat exchanger flow arrangement [1]

If algebraic expressions that correlate $\mathrm{P}, \mathrm{R}$ and $\Psi$ are found, the use of computers in the design and performance analysis of heat exchangers with complex flow arrangements could be facilitated, and the error associated with reading data from the P-R- $\Psi$ chart would be eliminated. In this case, the spreadsheet design and performance analysis of heat exchangers becomes relatively simple and straightforward, especially when the relevant algebraic expressions are appended to the spreadsheet as add-ins. The MS Excel environment allows the appendage of computer programmes written in Visual Basic for Applications (VBA), called MS Excel add-in tools [3].

Therefore, this paper presents polynomial expressions and a computer tool (MS Excel add-in) for obtaining the heat exchanger LMTD correction factor for ten selected heat exchanger configurations and flow arrangements. The approach facilitates computer-aided design analysis of engineering problems and would be useful to the design engineer, and also assist in the training of the student engineers $[4,5,6]$. The charted correction factors are interpolated using the Langrage interpolation scheme to obtain polynomial expressions for the various heat exchangers considered. This is then coded in the MS Excel environment as an add-in.

\section{PROBLEM FORMULATION AND SOLUTION}

The problem is to devise an automatic scheme for determining the LMTD correction factors for the popular complex heat exchangers; thus, eliminating the use of the correction factor chart, which is illustrated in Figure 1.

Using the Langrage polynomial interpolation scheme [7, 8], one obtains the following numerical schemes for the correction factor, $\psi$, as a function of the dimensionless temperature, $P$, and the ratio of water equivalents, $R$ :

$$
\Psi_{k}(P, R)=\sum_{i=0}^{n} \Psi_{k, i}\left[\prod_{\substack{j=0 \\ j \neq i}}^{n} \frac{R-R_{k, j}}{R_{k, i}-R_{k, j}}\right]
$$




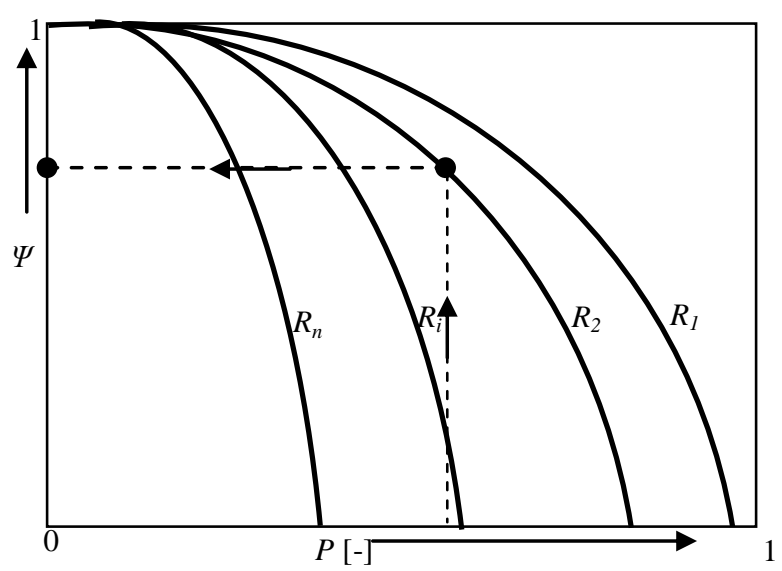

Figure 1 P-R- $\Psi$ chart for the determination of the LMTD correction factor $(\Psi)$

$P=\frac{T_{11}-T_{12}}{T_{11}-T_{21}}$

and

$$
R=\frac{\dot{C}_{1}}{\dot{C}_{2}}=\frac{T_{22}-T_{21}}{T_{11}-T_{12}}
$$

where $k$ is an integer value corresponding to the heat exchanger configuration type; and $n$ is the number of data points minus one; the first index $(i)$ in the double indexing scheme $(i j)$ stands for the hot $(i=1)$ or cold $(i=2)$ stream, while the second index $(j)$ stands for the heat exchanger inlet $(j=1)$ or exit $(j=2)$ temperature; that is: $\mathrm{T}_{11}$ is the hot stream inlet temperature; $T_{12}$ is the hot stream exit temperature; $T_{21}$ is the cold stream inlet temperature; and $\mathrm{T}_{22}$ is the cold stream exit temperature; and the water equivalent of the hot $(i=1)$ or cold $(i=2)$ stream, $\dot{C}_{i}$ is defined as

$$
\dot{C}_{i}=\dot{m}_{i} c_{p i} \quad[k W / K], i=1,2
$$

$\dot{m}_{i}[\mathrm{~kg} / \mathrm{s}]$ and $c_{p i}[\mathrm{~kJ} / \mathrm{kgK}]$ are the mass flow rate and isobaric specific heat capacity, respectively, for the hot $(i=1)$ or cold $(i=2)$ stream [1].

In determining the corrected logarithmic mean temperature difference (CLMTD), the counter-flow LMTD, $\Delta T_{L M C}$, is first obtained, which is given as (Oko, 2005)

$$
\Delta T_{L M C}=\frac{1}{A} \int_{0}^{A} \Delta T(A) d A=\frac{\Delta T_{b}-\Delta T_{s}}{\ln \frac{\Delta T_{b}}{\Delta T_{s}}}
$$

For the counter current flow, the temperature differences are given as follows:

(a) $\dot{C}_{1}>\dot{C}_{2}: \Delta T_{b}=T_{12}-T_{21}$ and $\Delta T_{s}=T_{11}-T_{22}$
(b) $\dot{C}_{1}<\dot{C}_{2}: \Delta T_{b}=T_{11}-T_{22}$ and $\Delta T_{s}=T_{12}-T_{21}$

where $\Delta T_{b}$ and $\Delta T_{s}$ are the big (b) and small (s) temperature differences, respectively, at the ends of the heat exchanger.

Therefore, the CLMTD becomes

$\Delta T_{C L M}=\psi \Delta T_{L M C}$

The heat transfer surface area $(A)$ is then determined from the fundamental equation of heat transfer as

$$
A=\frac{\dot{Q}}{U \Delta T_{C L M}}
$$

where $\dot{Q}[k W]$ is the heat transfer and $U\left[\mathrm{~kW} / \mathrm{m}^{2} \mathrm{~K}\right]$ is the overall heat transfer coefficient or the $U$-value.

The heat exchanger correction factor and CLMTD are determined using the following computational algorithm:

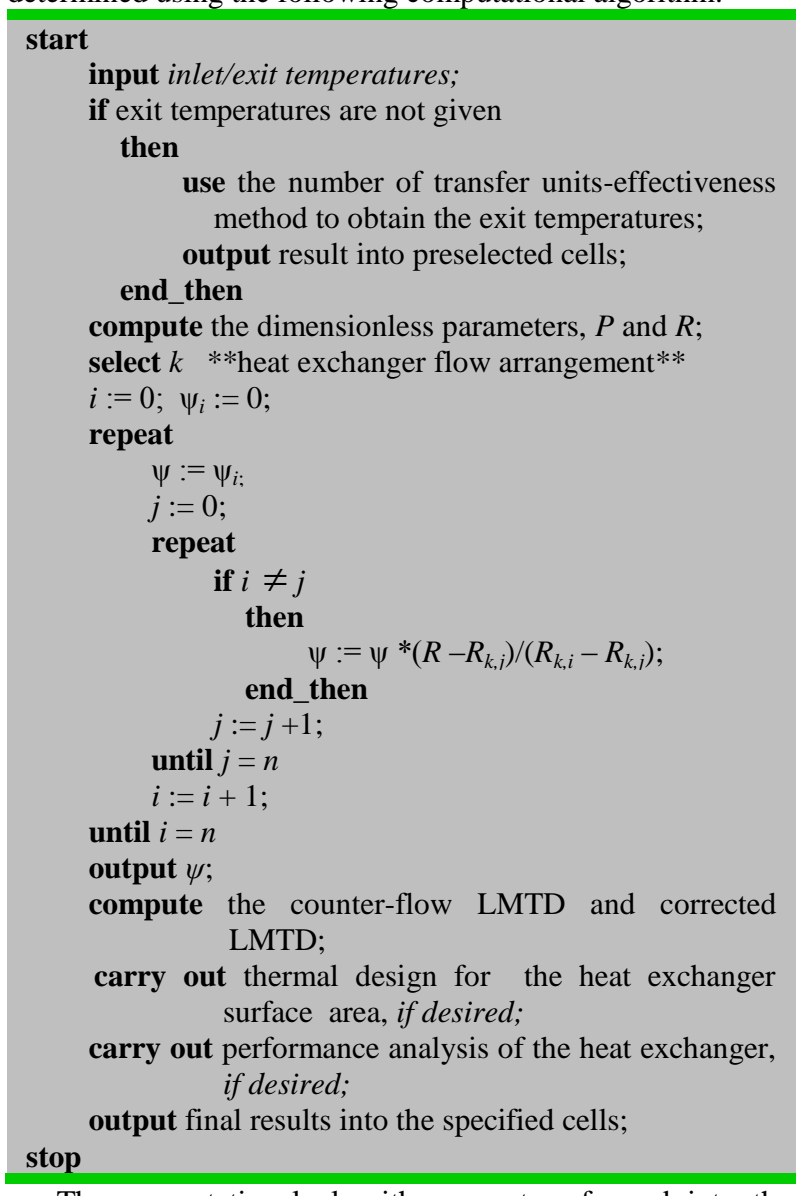

The computational algorithm was transformed into the Visual Basic for Application program in MS Excel as an addin tool.

\section{RESULTS AND DISCUSSION}

The MS Excel Solver is a popular computer tool for curve fitting data points when there is no direct tool of curve fitting $[3,9]$. The MS Excel Solver was used to curve fit the charted correction factors. The correlations for the correction factor, $\Psi$, as a function of the dimensionless temperature, $P$, at a specified ratio of the water equivalents of the two streams, $R$, in the interval $R \in[0.2,0.4]$ are presented in Table 1 , where $k$ is an integer value corresponding to the heat exchanger configuration type ( 1 shell pass, 2 or even number of tube passes (1-2n), 2 shell passes, 4 or multiple of 4 tube passes (2$4 n), 3$ shell passes 6 or multiple of 6 tube passes (3-6n), 4 shell passes 8 or multiple of 8 tube passes $(4-8 n), 5$ shell passes 10 or multiple of 10 tube passes (5-10n), 6 shell passes 12 or multiple of 12 tube passes (6-12n), Split flow shell 2 tube passes, 1 Divided flow shell pass even number of tube passes, Single pass cross flow both fluid unmixed, Single pass cross flow with one fluid mixed and the other unmixed), see the Appendix for schematic diagram of the configurations, and $i$ is the data point corresponding to the correction factor, $\psi$. 
Table 1 Correction Factor as a Function of Dimensionless Pressure and Heat Capacity Ratio

\begin{tabular}{|c|c|c|c|}
\hline$k$ & $i$ & Correction factor, $\Psi_{i}[-]$ & at $R_{i}[-]$ \\
\hline \multirow{13}{*}{0} & 0 & $-4.873 P^{3}+5.150 P^{2}-1.174 P+1.001$ & 0.200 \\
\hline & 1 & $-4.792 P^{3}+4.186 P^{2}-0.847 P+1.001$ & 0.400 \\
\hline & 2 & $-5.579 P^{3}+4.044 P^{2}-0.685 P+1.001$ & 0.600 \\
\hline & 3 & $-7.579 P^{3}+4.854 P^{2}-0.739 P+1.002$ & 0.800 \\
\hline & 4 & $-9.750 P^{3}+5.637 P^{2}-0.794 P+1.002$ & 1.000 \\
\hline & 5 & $-12.248 P^{3}-6.151 P^{2}+0.748 P+1.002$ & 1.200 \\
\hline & 6 & $-19.986 P^{3}-9.962 P^{2}+1.178 P+1.002$ & 1.400 \\
\hline & 7 & $-24.413 P^{3}-11.157 P^{2}-1.232 P+1.001$ & 1.600 \\
\hline & 8 & $-33.365 P^{3}+14.353 P^{2}-1.455 P+1.002$ & 1.800 \\
\hline & 9 & $-35.759 P^{3}+13.747 P^{2}-1.240 P+1.002$ & 2.000 \\
\hline & 10 & $-54.737 P^{3}+18.049 P^{2}-1.429 P+1.002$ & 2.500 \\
\hline & 11 & $-113.609 P^{3}+35.269 P^{2}-2.526 P+1.002$ & 3.000 \\
\hline & 12 & $-252.162 P^{3+} 65.926 P^{2}-4.068 P+1.001$ & 4.000 \\
\hline \multirow{13}{*}{1} & 0 & $-14.071 P^{3}+20.697 P^{2}-7.117 P+1.000$ & 0.200 \\
\hline & 1 & $-6.293 P^{3}+7.941 P^{2}-2.339 P+1.000$ & 0.400 \\
\hline & 2 & $-5.722 P^{3}+6.392 P^{2}-1.687 P+1.000$ & 0.600 \\
\hline & 3 & $-6.771 P^{3}+6.752 P^{2}-1.606 P+1.001$ & 0.800 \\
\hline & 4 & $-8.231 P^{3}+7.247 P^{2}-1.505 P+1.001$ & 1.000 \\
\hline & 5 & $-11.979 P^{3}+9.759 P^{2}-1.909 P+1.001$ & 1.200 \\
\hline & 6 & $-15.127 P^{3}+11.010 P^{2}-1.882 P+1.001$ & 1.400 \\
\hline & 7 & $-22.008 P^{3}+14.704 P^{2}-2.285 P+1.001$ & 1.600 \\
\hline & 8 & $-35.076 P^{3}+22.312 P^{2}-3.327 P+1.000$ & 1.800 \\
\hline & 9 & $-34.844 P^{3}+19.400 P^{2}-2.426 P+1.001$ & 2.000 \\
\hline & 10 & $-68.328 P^{3}+32.857 P^{2}-3.595 P+1.001$ & 2.500 \\
\hline & 11 & $-145.351 P^{3}+58.871 P^{2}-5.109 P+1.000$ & 3.000 \\
\hline & 12 & $-403.127 P^{3}+131.391 P^{2}-9.363 P+1.000$ & 4.000 \\
\hline \multirow{13}{*}{2} & 0 & $-43.441 P^{3}+70.811 P^{2}-27.789 P+1.001$ & 0.200 \\
\hline & 1 & $-14.738 P^{3}+22.121 P^{2}-7.935 P+1.000$ & 0.400 \\
\hline & 2 & $-8.770 P^{3}+11.459 P^{2}-3.468 P+1.000$ & 0.600 \\
\hline & 3 & $-7.657 P^{3}+9.153 P^{2}-2.614 P+1.000$ & 0.800 \\
\hline & 4 & $-9.036 P^{3}+9.599 P^{2}-2.431 P+1.000$ & 1.000 \\
\hline & 5 & $-12.156 P^{3}+11.775 P^{2}-2.743 P+1.000$ & 1.200 \\
\hline & 6 & $-19.838 P^{3}+17.933 P^{2}-3.931 P+1.000$ & 1.400 \\
\hline & 7 & $-27.599 P^{3}+22.345 P^{2}-4.326 P+1.000$ & 1.600 \\
\hline & 8 & $-47.145 P^{3}+35.919 P^{2}-6.578 P+1.000$ & 1.800 \\
\hline & 9 & $-67.481 P^{3}+46.527 P^{2}-7.601 P+1.000$ & 2.000 \\
\hline & 10 & $-143.027 P^{3}+83.130 P^{2}-11.490 P+1.000$ & 2.500 \\
\hline & 11 & $-552.940 P^{3}+270.110 P^{2}-30.175 P+0.999$ & 3.000 \\
\hline & 12 & $-869.914 P^{3}+316.038 P^{2}-26.012 P+1.000$ & 4.000 \\
\hline \multirow{13}{*}{3} & 0 & $-71.544 P^{3}+124.149 P^{2}-53.014 P+1.050$ & 0.200 \\
\hline & 1 & $-32.605 P^{3}+52.519 P^{2}-20.426 P+0.997$ & 0.400 \\
\hline & 2 & $-14.241 P^{3}+21.245 P^{2}-7.642 P+1.000$ & 0.600 \\
\hline & 3 & $-9.717 P^{3}+12.998 P^{2}-4.200 P+1.000$ & 0.800 \\
\hline & 4 & $-11.390 P^{3}+13.552 P^{2}-3.854 P+1.000$ & 1.000 \\
\hline & 5 & $-22.886 P^{3}+25.458 P^{2}-6.756 P+1.000$ & 1.200 \\
\hline & 6 & $-25.985 P^{3}+25.754 P^{2}-6.151 P+1.000$ & 1.400 \\
\hline & 7 & $-46.027 P^{3}+42.168 P^{2}-9.382 P+1.000$ & 1.600 \\
\hline & 8 & $-78.195 P^{3}+66.440 P^{2}-13.768 P+1.000$ & 1.800 \\
\hline & 9 & $-163.035 P^{3}+128.648 P^{2}-24.656 P+1.000$ & 2.000 \\
\hline & 10 & $-315.336 P^{3}+194.804 P^{2}-28.505 P+1.002$ & 2.500 \\
\hline & 11 & $-1737.783 P^{3}+941.669 P^{2}-122.693 P+1.0$ & 3.000 \\
\hline & 12 & $-1819.250 P^{3}+755.072 P^{2}-76.400 P+1.0$ & 4.000 \\
\hline \multirow{12}{*}{4} & 0 & $-654.453 P^{3}+1188.742 P^{2}-534.943 P+1.0$ & 0.200 \\
\hline & 1 & $-53.370 P^{3}+92.083 P^{2}-39.081 P+0.980$ & 0.400 \\
\hline & 2 & $-25.602 P^{3}+41.358 P^{2}-16.335 P+1.000$ & 0.600 \\
\hline & 3 & $-14.871 P^{3}+21.933 P^{2}-7.918 P+1.000$ & 0.800 \\
\hline & 4 & $-16.143 P^{3}+21.395 P^{2}-6.916 P+1.000$ & 1.000 \\
\hline & 5 & $-23.106 P^{3}+27.637 P^{2}-8.087 P+0.999$ & 1.200 \\
\hline & 6 & $-37.198 P^{3}+39.555 P^{2}-10.191 P+0.999$ & 1.400 \\
\hline & 7 & $-83.785 P^{3}+81.898 P^{2}-19.395 P+0.999$ & 1.600 \\
\hline & 8 & $-131.687 P^{3}+116.690 P^{2}-25.046 P+1.000$ & 1.800 \\
\hline & 9 & $-167.264 P^{3}+134.884 P^{2}-26.314 P+0.998$ & 2.000 \\
\hline & 10 & $-534.182 P^{3}+357.030 P^{2}-58.185 P+0.996$ & 2.500 \\
\hline & 11 & $-2254.758 P^{3}+1270.159 P^{2}-175.063 P+$ & 3.000 \\
\hline
\end{tabular}

\begin{tabular}{|c|c|c|c|}
\hline \multirow[t]{3}{*}{$k$} & $i$ & Correction factor, $\Psi_{i}[-]$ & at $R_{i}[-]$ \\
\hline & & 1.035 & \\
\hline & 12 & $\begin{array}{c}-2393.321 P^{3}+937.029 P^{2}-91.010 P+ \\
0.999\end{array}$ & 4.000 \\
\hline \multirow{13}{*}{5} & 0 & $-837.062 P^{3}+1522.927 P^{2}-686.254 P+1.0$ & 0.200 \\
\hline & 1 & $\begin{array}{l}-870.687 P^{3}+1518.984 P^{2}-649.177 P+ \\
1.030\end{array}$ & 0.400 \\
\hline & 2 & $-21.880 P^{3}+34.798 P^{2}-13.364 P+1.002$ & 0.600 \\
\hline & 3 & $-13.806 P^{3}+20.116 P^{2}-7.024 P+1.000$ & 0.800 \\
\hline & 4 & $-16.852 P^{3}+22.788 P^{2}-7.438 P+1.000$ & 1.000 \\
\hline & 5 & $-22.930 P^{3}+28.363 P^{2}-8.556 P+1.000$ & 1.200 \\
\hline & 6 & $-55.218 P^{3}+62.759 P^{2}-17.476 P+0.998$ & 1.400 \\
\hline & 7 & $-87.778 P^{3}+86.820 P^{2}-20.812 P+1.001$ & 1.600 \\
\hline & 8 & $-196.828 P^{3}+179.023 P^{2}-39.479 P+0.999$ & 1.800 \\
\hline & 9 & $-207.755 P^{3}+167.185 P^{2}-32.382 P+1.000$ & 2.000 \\
\hline & 10 & $-1353.721 P^{3}+921.755 P^{2}-153.134 P+1.0$ & 2.500 \\
\hline & 11 & $-143.501 P^{3}+71.178 P^{2}-8.847 P+1.000$ & 3.000 \\
\hline & 12 & $\begin{array}{c}-7933.555 P^{3}+3147.121 P^{2}-307.820 P+ \\
1.018\end{array}$ & 4.000 \\
\hline \multirow{13}{*}{6} & 0 & $-15.989 P^{3}+23.415 P^{2}-7.886 P+1.000$ & 0.200 \\
\hline & 1 & $-4.929 P^{3}+6.262 P^{2}-1.845 P+1.000$ & 0.400 \\
\hline & 2 & $-5.127 P^{3}+5.711 P^{2}-1.452 P+1.000$ & 0.600 \\
\hline & 3 & $-5.534 P^{3}+5.331 P^{2}-1.187 P+1.001$ & 0.800 \\
\hline & 4 & $-7.221 P^{3}+6.161 P^{2}-1.239 P+1.001$ & 1.000 \\
\hline & 5 & $-12.580 P^{3}+10.178 P^{2}-1.924 P+1.001$ & 1.200 \\
\hline & 6 & $-16.235 P^{3}+11.849 P^{2}-2.051 P+1.001$ & 1.400 \\
\hline & 7 & $-25.656 P^{3}+17.450 P^{2}-2.759 P+1.000$ & 1.600 \\
\hline & 8 & $-35.166 P^{3}+21.721 P^{2}-3.085 P+1.000$ & 1.800 \\
\hline & 9 & $-45.298 P^{3}+25.728 P^{2}-3.337 P+1.000$ & 2.000 \\
\hline & 10 & $-82.821 P^{3}+39.542 P^{2}-4.289 P+1.000$ & 2.500 \\
\hline & 11 & $-153.910 P^{3}+63.335 P^{2}-5.831 P+1.000$ & 3.000 \\
\hline & 12 & $-373.251 P^{3}+119.218 P^{2}-8.194 P+1.000$ & 4.000 \\
\hline \multirow{13}{*}{7} & 0 & $-3.291 P^{3}+3.276 P^{2}-0.704 P+1.000$ & 0.200 \\
\hline & 1 & $-3.313 P^{3}+2.644 P^{2}-0.507 P+1.000$ & 0.400 \\
\hline & 2 & $-4.030 P^{3}+2.643 P^{2}-0.422 P+1.000$ & 0.600 \\
\hline & 3 & $-5.049 P^{3}+2.795 P^{2}-0.390 P+1.000$ & 0.800 \\
\hline & 4 & $-6.567 P^{3}+3.213 P^{2}-0.399 P+1.000$ & 1.000 \\
\hline & 5 & $-9.019 P^{3}+4.130 P^{2}-0.477 P+1.000$ & 1.200 \\
\hline & 6 & $-14.599 P^{3}+6.666 P^{2}-0.752 P+1.000$ & 1.400 \\
\hline & 7 & $-18.155 P^{3}+7.501 P^{2}-0.760 P+1.000$ & 1.600 \\
\hline & 8 & $-25.262 P^{3}+10.220 P^{2}-1.003 P+1.000$ & 1.800 \\
\hline & 9 & $-26.688 P^{3}+9.146 P^{2}-0.734 P+1.000$ & 2.000 \\
\hline & 10 & $-46.146 P^{3}+13.684 P^{2}-0.857 P+1.001$ & 2.500 \\
\hline & 11 & $-68.293 P^{3}+16.852 P^{2}-0.791 P+1.001$ & 3.000 \\
\hline & 12 & $-180.183 P^{3}+38.179 P^{2}-1.398 P+1.001$ & 4.000 \\
\hline \multirow{8}{*}{8} & 0 & $-4.070 P^{3}+4.645 P^{2}-1.025 P+1.001$ & 0.200 \\
\hline & 1 & $-2.003 P^{3}+1.897 P^{2}-0.407 P+1.002$ & 0.400 \\
\hline & 2 & $-1.660 P^{3}+1.277 P^{2}-0.261 P+1.002$ & 0.600 \\
\hline & 3 & $-1.283 P^{3}+0.583 P^{2}-0.080 P+1.001$ & 0.800 \\
\hline & 4 & $-1.611 P^{3}+0.590 P^{2}-0.061 P+1.000$ & 1.000 \\
\hline & 5 & $-13.493 P^{3}+5.763 P^{2}-0.616 P+1.004$ & 2.000 \\
\hline & 6 & $-47.436 P^{3}+15.322 P^{2}-1.253 P+1.006$ & 3.000 \\
\hline & 7 & $-182.697 P^{3}+47.418 P^{2}-2.278 P+1.002$ & 4.000 \\
\hline \multirow{8}{*}{9} & 0 & $-7.799 P^{3}+9.502 P^{2}-2.761 P+1.000$ & 0.200 \\
\hline & 1 & $-5.543 P^{3}+5.247 P^{2}-1.165 P+1.001$ & 0.400 \\
\hline & 2 & $-5.550 P^{3}+4.129 P^{2}-0.678 P+1.001$ & 0.600 \\
\hline & 3 & $-6.026 P^{3}+3.759 P^{2}-0.508 P+1.002$ & 0.800 \\
\hline & 4 & $-6.512 P^{3}+3.464 P^{2}-0.437 P+1.004$ & 1.000 \\
\hline & 5 & $-22.710 P^{3}+8.592 P^{2}-0.678 P+1.004$ & 2.000 \\
\hline & 6 & $-54.417 P^{3}+15.318 P^{2}-0.932 P+1.005$ & 3.000 \\
\hline & 7 & $-259.538 P^{3}+60.180 P^{2}-1.633 P+1.003$ & 4.000 \\
\hline
\end{tabular}

To obtain the correction factor $(\Psi)$ one substitutes $P$ into the correlations in Tables 1 that correspond to the heat exchanger flow arrangement under consideration and interpolates for $\Psi$ with $R$ serving as the interpolation point. 


\subsection{Examples}

As illustrations of the use of the developed spreadsheet add-in to determine the correction factors and other thermal design parameters for heat exchangers, the following three examples are provided:

\subsubsection{Example 1: Determination of the Correction Factors}

Use the data tabulated in Table 2 to determine the correction factors for the various shell-tube heat exchanger configurations [10].

\section{Table 1 Input Data for Example 2}

\begin{tabular}{|c|c|c|c|c|}
\hline S/No & Quantity & Symbol & Units & Value \\
\hline 1 & $\begin{array}{l}\text { tube side inlet } \\
\text { temperature of } \\
\text { the hot stream }\end{array}$ & $\mathrm{t}_{11}$ & ${ }^{\circ} \mathrm{C}$ & 80.00 \\
\hline 2 & $\begin{array}{l}\text { tube side exit } \\
\text { temperature of } \\
\text { the hot stream }\end{array}$ & $t_{12}$ & ${ }^{\circ} \mathrm{C}$ & 40.00 \\
\hline 3 & $\begin{array}{l}\text { shell side inlet } \\
\text { temperature of } \\
\text { the cold stream }\end{array}$ & $\mathrm{t}_{21}$ & ${ }^{\circ} \mathrm{C}$ & 20.00 \\
\hline 4 & $\begin{array}{l}\text { shell side exit } \\
\text { temperature of } \\
\text { the cold stream }\end{array}$ & $t_{22}$ & ${ }^{\circ} \mathrm{C}$ & 50.00 \\
\hline
\end{tabular}

\section{Solution}

Key in the developed MS Excel formula, $=\mathrm{HCF}(k, \mathrm{~A}, \mathrm{~B}, \mathrm{C}$, $\mathrm{D})$, in any desired MS Excel cell; where $k, \mathrm{~A}, \mathrm{~B}, \mathrm{C}$ and D are cell references holding numerical values of the integer $(k)$ that corresponds to the heat exchanger flow arrangement, tube side inlet temperature (A), tube side exit temperature (B), shell side inlet temperature (C) and shell side outlet temperature (D), respectively; holding down ctrl+shift keys, highlighting the four adjacent cells and pressing the enter key output the results shown in Table 3, which tabulates, among others, the dimensionless parameters $\mathrm{P}$ and $\mathrm{R}$, the correction factor $(\psi)$ and the corrected LMTD (CLMTD).

\section{Table 3 MS Excel Add-in Output Data for Example 1}

\begin{tabular}{|c|c|c|c|c|c|}
\hline Heat Exchanger Class & $P$ & $R$ & $\Psi$ & $\begin{array}{l}\text { LMTD } \\
{\left[{ }^{\circ} \mathrm{C}\right]}\end{array}$ & $\begin{array}{l}\text { CLMTD } \\
{\left[{ }^{\circ} \mathrm{C}\right]} \\
\end{array}$ \\
\hline $\begin{array}{l}1 \text { shell pass, } 2 \text { or even } \\
\text { number of tube passes }\end{array}$ & 0.67 & 0.75 & 0.540 & 24.66 & 13.31 \\
\hline $\begin{array}{l}2 \text { shell passes, } 4 \text { or multiple } \\
\text { of } 4 \text { tube passes }\end{array}$ & 0.67 & 0.75 & 0.911 & 24.66 & 22.48 \\
\hline $\begin{array}{l}3 \text { shell passes, } 6 \text { or multiple } \\
\text { of } 6 \text { tube passes }\end{array}$ & 0.67 & 0.75 & 0.962 & 24.66 & 23.73 \\
\hline $\begin{array}{l}4 \text { shell passes, } 8 \text { or multiple } \\
\text { of } 8 \text { tube passes. }\end{array}$ & 0.67 & 0.75 & 0.979 & 24.66 & 24.15 \\
\hline $\begin{array}{l}5 \text { shell passes, } 10 \text { or multiple } \\
\text { of } 10 \text { tube passes }\end{array}$ & 0.67 & 0.75 & 0.987 & 24.66 & 24.34 \\
\hline $\begin{array}{l}6 \text { shell passes, } 12 \text { or multiple } \\
\text { of } 12 \text { tube passes }\end{array}$ & 0.67 & 0.75 & 0.991 & 24.66 & 24.44 \\
\hline $\begin{array}{l}\text { Split flow shell, } 2 \text { tube } \\
\text { passes }\end{array}$ & 0.67 & 0.75 & 0.951 & 24.66 & 23.45 \\
\hline $\begin{array}{l}1 \text { Divided flow shell pass, } \\
\text { even number of tube passes }\end{array}$ & 0.67 & 0.75 & 0.573 & 24.66 & 14.13 \\
\hline $\begin{array}{l}\text { Single pass cross flow both } \\
\text { fluid unmixed }\end{array}$ & 0.67 & 0.75 & 0.629 & 24.66 & 15.51 \\
\hline $\begin{array}{l}\text { Single pass cross flow with } \\
\text { one fluid mixed and the } \\
\text { other unmixed }\end{array}$ & 0.67 & 0.75 & 0.700 & 24.66 & 17.27 \\
\hline
\end{tabular}

The counter-flow $L M T D$ for the problem is constant, $L M T D_{c}=$ $24.66\left[{ }^{\circ} \mathrm{C}\right]$, which is higher than any of the CLMTD of the heat exchanger configurations considered. With the use of the $L M T D_{c}$ value, one would have under-designed the heat exchangers because less surface area would be provided, which would not be able to match the intended heat transfer load. The results show that for the six types of shell and tube heat exchangers considered, the CLMTD value increases progressively with the number of shell passes, which means that given the same heat transfer surface area U-value, the effectiveness of the shell and tube heat exchangers increases with increasing numbers of shell passes. Cengel [10] reported 0.92 correction factor read from chart for 2 shell passes, 4 or multiple of 4 tube passes with the same input data, which is about $1.0 \%$ deviation from that obtained with the spreadsheet add-in. Although this deviation is acceptable for most engineering applications, it cannot be directly traced to the approach adopted in this study, since reading data from charts as Cengel [10] is always prone to errors.

\subsubsection{Example 2: Determination of the Heat Exchanger Surface Area}

$45.4 \mathrm{~kg} / \mathrm{h}$ of water is to be heated from 10 to $77^{\circ} \mathrm{C}$ with flue gases having an initial temperature of $166^{\circ} \mathrm{C}$. The mass flow rate of the gases is $182 \mathrm{~kg} / \mathrm{h}$ and their specific heat is $1.05 \mathrm{~kJ} / \mathrm{kgK}$. The overall heat transfer coefficient may be taken as $114 \mathrm{~W} / \mathrm{m}^{2} \mathrm{~K}$. Calculate the size of the heating surface for a 1 - 2 shell-tube heat exchanger [1].

\section{Solution}

Input data:

$\dot{m}_{w}=0.0126 \mathrm{~kg} / \mathrm{s} ; \mathrm{t}_{21}=10{ }^{\circ} \mathrm{C} ; \mathrm{t}_{22}=77{ }^{\circ} \mathrm{C} ; \mathrm{t}_{11}=166{ }^{\circ} \mathrm{C} ; \dot{m}_{g}$ $=0.0506 \mathrm{~kg} / \mathrm{s} ; c_{p g}=1.05 \mathrm{~kJ} / \mathrm{kgK} ; \mathrm{U}=0.114 \mathrm{~kW} / \mathrm{m}^{2} \mathrm{~K} ; \mathrm{c}_{\mathrm{pw}}=$ $4.18 \mathrm{~kJ} / \mathrm{kgK} ; 1-2$ shell-tube heat exchanger;

The results obtained with the spreadsheet add-in are shown in Table 4. The results indicate that with the use of only the counter-flow LMTD for the design would lead to under-designing the heat exchanger surface area by $10.21 \%$.

Table 4 MS Excel Add-in Output Data for Example 2

\begin{tabular}{|c|c|c|c|c|c|}
\hline & Quantity & Symbol & Units & Formula & Value \\
\hline & $\begin{array}{l}\text { heat capacity } \\
\text { ratio }\end{array}$ & $\omega$ & - & $\omega=\dot{m}_{w} * \mathrm{c}_{\mathrm{pw}} /\left(\mathrm{m}_{\mathrm{g}} * \mathrm{c}_{\mathrm{pg}}\right)$ & 0.995 \\
\hline & $\begin{array}{l}\text { flue gases exit } \\
\text { temperature }\end{array}$ & $t_{12}$ & ${ }^{\circ} \mathrm{C}$ & $\mathrm{t}_{12}=\mathrm{t}_{11}-\omega^{*}\left(\mathrm{t}_{22}-\mathrm{t}_{21}\right)$ & 99.31 \\
\hline 3 & $\begin{array}{l}\text { counter flow big } \\
\text { temperature } \\
\text { difference }\end{array}$ & $\Delta \mathrm{t}_{\mathrm{b}}$ & ${ }^{\circ} \mathrm{C}$ & $\Delta \mathrm{t}_{\mathrm{b}}=\mathrm{t}_{11-}-\mathrm{t}_{22}$ & 89.00 \\
\hline 4 & $\begin{array}{l}\text { counter flow } \\
\text { small } \\
\text { temperature } \\
\text { difference }\end{array}$ & $\Delta t_{\mathrm{s}}$ & ${ }^{\circ} \mathrm{C}$ & $\Delta \mathrm{t}_{\mathrm{s}}=\mathrm{t}_{12-}-\mathrm{t}_{21}$ & 89.31 \\
\hline 5 & $\begin{array}{l}\text { counter flow } \\
\text { LMTD, LMTD }_{C}\end{array}$ & $\Delta \mathrm{t}_{\mathrm{LMC}}$ & ${ }^{\circ} \mathrm{C}$ & $=\operatorname{HCF}\left(0, \mathrm{t}_{11}, \mathrm{t}_{12}, \mathrm{t}_{21}, \mathrm{t}_{22}\right)$ & 89.15 \\
\hline 6 & $\begin{array}{l}\text { heat exchanger } \\
\text { correction factor }\end{array}$ & $\Psi$ & - & $=\operatorname{HCF}\left(0, \mathrm{t}_{11}, \mathrm{t}_{12}, \mathrm{t}_{21}, \mathrm{t}_{22}\right)$ & 0.898 \\
\hline 7 & $\begin{array}{l}\text { corrected } \\
\text { LMTD, } \\
\text { CLMTD }\end{array}$ & $\Delta \mathrm{t}_{\mathrm{CLM}}$ & ${ }^{\circ} \mathrm{C}$ & $=\operatorname{HCF}\left(0, \mathrm{t}_{11}, \mathrm{t}_{12}, \mathrm{t}_{21}, \mathrm{t}_{22}\right)$ & 80.06 \\
\hline 8 & $\begin{array}{l}\text { Heat transfer } \\
\text { rate }\end{array}$ & $\dot{Q}$ & $\mathrm{~kW}$ & $\dot{Q}=\dot{m}_{w} * \mathrm{c}_{\mathrm{pw}} *\left(\mathrm{t}_{22}-\mathrm{t}_{21}\right)$ & 3.540 \\
\hline 9 & $\begin{array}{l}\text { Heat transfer } \\
\text { surface area } \\
\text { without } \\
\text { correction factor }\end{array}$ & $\mathrm{A}_{\mathrm{cf}}$ & $\mathrm{m}^{2}$ & $\mathrm{~A}_{\mathrm{cf}}=\dot{Q}^{/\left(\mathrm{U}^{*} \Delta \mathrm{t}_{\mathrm{LMC}}\right)}$ & 0.3483 \\
\hline 10 & $\begin{array}{l}\text { Heat transfer } \\
\text { surface area } \\
\text { with correction } \\
\text { factor }\end{array}$ & A & $\mathrm{m}^{2}$ & $\mathrm{~A}=\dot{Q} /\left(\mathrm{U}^{*} \Delta \mathrm{t}_{\mathrm{CLM}}\right)$ & 0.3879 \\
\hline 11 & $\begin{array}{l}\text { Percentage } \\
\text { change in heat } \\
\text { transfer surface } \\
\text { area }\end{array}$ & $\varepsilon$ & $\%$ & $\varepsilon=\left(\left(\mathrm{A}-\mathrm{A}_{\mathrm{cf}}\right) / \mathrm{A}\right)^{*} * 100$ & 10.21 \\
\hline
\end{tabular}


3.1.3 Example 3: Determination of Exit Temperatures, Correction Factors and Heat Transfer Rates

Use the flow and fluid properties of the six heat exchangers, HX1- HX5 [11] and HX6 [10], shown in Table 5 to carry out thermal analysis of the given six heat exchangers.

Table 5 Input Data for the Six Heat Exchangers, Example 3

\begin{tabular}{|c|c|c|c|c|c|c|}
\hline \multirow{3}{*}{$\begin{array}{l}\text { Flow and Fluid } \\
\text { Properties }\end{array}$} & \multicolumn{6}{|c|}{ Heat Exchanger } \\
\hline & \multicolumn{2}{|c|}{ HX1 } & \multicolumn{2}{|c|}{ HX2 } & \multicolumn{2}{|l|}{ HX3 } \\
\hline & Tube & Shell & Tube & Shell & Tube & Shell \\
\hline heat capacity, & 2.05 & 2.47 & 2.47 & 2.05 & 2.05 & 2.28 \\
\hline $\begin{array}{l}\text { mass flow rate, } \\
\dot{m}, \mathrm{~kg} / \mathrm{s} \text {. }\end{array}$ & 116.1 & 32.3 & 125.5 & 232.3 & 116.1 & 10.9 \\
\hline $\begin{array}{l}\text { inlet } \\
\text { temperature, }\end{array}$ & 441 & 365 & 457 & 370.1 & 581 & 382 \\
\hline $\begin{array}{l}\mathrm{t}_{11}, \mathrm{t}_{21},{ }^{\circ} \mathrm{C} . \\
\text { number } \\
\text { passes. }\end{array}$ & 2 & 1 & 2 & 1 & 2 & 1 \\
\hline $\begin{array}{l}\text { heat transfer } \\
\text { surface area, } \mathrm{A} \text {, } \\
\mathrm{m}^{2} \text {. }\end{array}$ & \multicolumn{2}{|c|}{418} & \multicolumn{2}{|c|}{379} & \multicolumn{2}{|c|}{327} \\
\hline $\begin{array}{l}\text { heat transfer } \\
\text { coefficient, U, } \\
\mathrm{kW} / \mathrm{m}^{2} \mathrm{~K} \text {. }\end{array}$ & \multicolumn{2}{|c|}{0.3033} & \multicolumn{2}{|c|}{0.3916} & \multicolumn{2}{|c|}{0.1737} \\
\hline
\end{tabular}

\section{Table 5 Continues}

\begin{tabular}{|c|c|c|c|c|c|c|}
\hline \multirow{3}{*}{$\begin{array}{l}\text { Flow and Fluid } \\
\text { Properties }\end{array}$} & \multicolumn{6}{|c|}{ Heat Exchanger } \\
\hline & \multicolumn{2}{|c|}{ HX4 } & \multicolumn{2}{|c|}{ HX5 } & \multicolumn{2}{|l|}{ HX6 } \\
\hline & Tube & Shell & Tube & Shell & Tube & Shell \\
\hline $\begin{array}{l}\text { heat capacity, } \\
c_{\mathrm{p}}, \mathrm{kJ} / \mathrm{kgK} \text {. }\end{array}$ & 2.05 & 2.28 & 2.05 & 2.47 & 4.18 & 2.13 \\
\hline $\begin{array}{l}\text { mass flow rate, } \\
\dot{m}, \mathrm{~kg} / \mathrm{s} \text {. }\end{array}$ & 116.1 & 56.0 & 232.3 & 32.2 & 0.2 & 0.3 \\
\hline $\begin{array}{l}\text { inlet } \\
\text { temperature, }\end{array}$ & 365 & 480 & 506 & 431 & 20 & 150 \\
\hline $\begin{array}{l}t_{11}, 2_{21}, \mathrm{C} . \\
\text { number } \\
\text { passes. }\end{array}$ & 2 & 1 & 2 & 1 & 8 & 1 \\
\hline $\begin{array}{l}\text { heat transfer } \\
\text { surface area, } A, \\
\mathrm{~m}^{2} \text {. }\end{array}$ & & 18 & & & 1.76 & \\
\hline $\begin{array}{l}\text { heat transfer } \\
\text { coefficient, U, } \\
\mathrm{kW} / \mathrm{m}^{2} \mathrm{~K} \text {. }\end{array}$ & & & & 928 & 0.31 & \\
\hline
\end{tabular}

\section{Solution}

Figure 2 shows the sketch of temperature distribution along any of the heat exchangers.

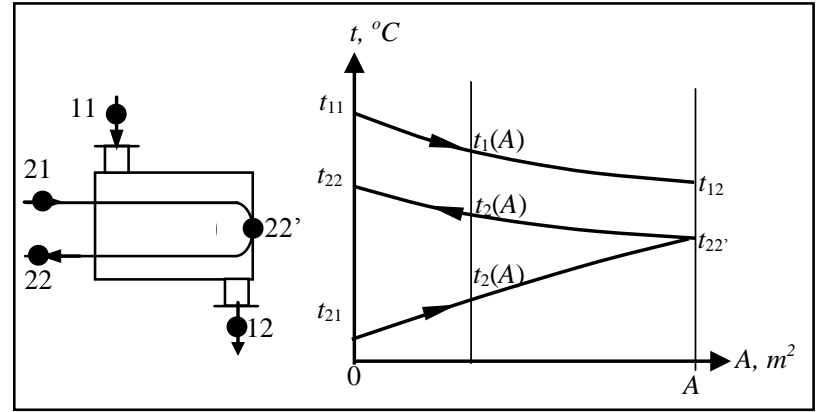

Figure 2 Sketch/Diagram of any of the Heat Exchangers

The heat exchanger performance analysis is carried out as follows:

(i) obtain the exit temperatures, $\mathrm{T}_{12}$ and $\mathrm{T}_{22}$ by keying in the MS Excel user defined function (add-in), $=$ EFFNTU2 $(\mathrm{A}, \mathrm{B}, \mathrm{C}, \mathrm{D}, \mathrm{E}, \mathrm{F}, \mathrm{G})$ in any desired cell, where A, B, C, D, E, F and G stand for error bound, heat transfer coefficient, inlet temperature of the hot stream; inlet temperature of the cold stream, water equivalent of the hot stream, water equivalent of the cold stream and the flow configuration, respectively;

(ii) use the function $\operatorname{HCF}(\mathrm{k}, \mathrm{H}, \mathrm{I}, \mathrm{J}, \mathrm{K})$ to obtain the counter-flow LMTD, correction factor and corrected LMTD; and

(iii) with the corrected LMTD obtained, compute the heat transfer rate.

The results of the thermal analysis are shown in Table 6 . Cengel [10] provided results for heat exchanger HX6, which are in agreement with results for HX6 as shown in Table 6. HX4 bears the largest thermal load, and the lowest thermal load is borne by HX6. The reason is attributed to the largest and lowest values of the product of overall heat transfer coefficient and heat transfer surface area for heat exchanger HX4 and HX6, respectively. The exit temperatures for both streams are highest in HX3, which is attributed to the mass flow rates, heat capacities and inlet temperatures of the fluid streams.

Table 6 MS Excel Add-in Output data for Example 3

\begin{tabular}{l|cc|cc|cc}
\hline \multirow{2}{*}{ Quantity } & \multicolumn{5}{|c}{ Heat Exchanger } \\
\cline { 2 - 7 } & \multicolumn{2}{|c|}{ HX1 } & \multicolumn{2}{c}{ HX2 } & \multicolumn{2}{c}{ HX3 } \\
\cline { 2 - 6 } & Tube & Shell & Tube & Shell & Tube & Shell \\
\hline water equivalent, & 238.0 & 79.8 & 310.0 & 476.2 & 238.0 & 24.9 \\
$\dot{C}, \mathrm{~kW} / \mathrm{K}$ & & & & & & \\
exit & 423.3 & 417.7 & 429.9 & 387.7 & 563.8 & 547.2 \\
temperatures, & & & & & & \\
$\mathrm{T}_{12}, \mathrm{~T}_{22}, \mathrm{~K}$ & & & & & \\
correction factor & 0.85 & 0.92 & 0.99 \\
counter-flow & 38.20 & 64.40 & 87.98 \\
$\begin{array}{c}\text { LMTD } \\
\text { corrected LMTD }\end{array}$ & 32.47 & 59.25 & 87.1 \\
heat transfer rate, & 4116.64 & 8793.84 & 4947.36 \\
Q, kW & & & & \\
\hline
\end{tabular}


Table 6 Continues

\begin{tabular}{l|cc|cc|cc}
\hline \multirow{2}{*}{ Quantity } & \multicolumn{5}{c}{ Heat Exchanger } \\
\cline { 2 - 7 } & \multicolumn{2}{|c|}{ HX4 } & \multicolumn{2}{c}{ HX5 } & \multicolumn{2}{c}{ HX6 } \\
\cline { 2 - 6 } & Tube & Shell & Tube & Shell & Tube & Shell \\
\hline $\begin{array}{l}\text { water equivalent, } \\
\dot{C}, \mathrm{~kW} / \mathrm{K}\end{array}$ & 238.0 & 127.7 & 476.2 & 79.54 & 0.84 & 0.64 \\
$\begin{array}{c}\text { exit } \\
\text { temperatures, }\end{array}$ & 398.9 & 416.8 & 496.9 & 485.8 & 66.2 & 89.6 \\
$\mathrm{~T}_{12}, \mathrm{~T}_{22}, \mathrm{~K}$ & & & & & & \\
correction factor \\
$\begin{array}{c}\text { counter-flow } \\
\text { LMTD }\end{array}$ & 0.98 & & 0.78 & 0.98 \\
corrected LMTD \\
heat transfer rate, \\
\begin{tabular}{l}
$\mathrm{Q}, \mathrm{kW}$ \\
\hline
\end{tabular}
\end{tabular}

\section{CONCLUSION}

An MS Excel add-in for heat exchanger LMTD correction factor has been developed for ten popular heat exchanger configurations, $k=0,1, \ldots, 9$. It is a tool for direct computation of the LMTD correction factors, which eliminates the existing cumbersome graphical and iterative methods [12]. Results obtained are accurate enough for engineering applications. Several examples have been used to demonstrate the utility of the add-in tool, which is user friendly (interactive), robust and flexible. Apart from the stand-alone application of this spreadsheet add-in tool, it can also be integrated into a larger plant design software for improved productivity. Of course, the add-in is also a veritable tool for the effective teaching of the thermal design of heat exchangers in higher institutions of learning $[6,13]$. The spreadsheet addin if integrated into a larger plant can also be used by practicing engineers for heat exchanger design, simulation and selection.

\section{REFERENCES}

[1] Oko, C.O.C. 2005. Introductory Heat Transfer: An algorithmic approach, $2^{\text {nd }}$ Edition, Pam Unique publishing company limited, Port Harcourt.

[2] Bell, J.K. and Mueller, C.A. 2001. Wolverine engineering data Book II, Wolverine tube Inc., www.wlv.com/products/databook/ch2_2.pdf, Retrieved: 28/03/10.

[3] Liengme, B.V. (2000) A Guide to Microsoft Excel for scientist and engineers, Butterworth-Heinemann, London.

[4] Oko, C.O.C., Diemuodeke, E.O. and Akinlade, I.S. 2010. Design of hoppers using spreadsheet, Journal of Research in Agricultural Engineering, Vol. 56(2), pp. 53-58.

[5] Lona, L.M.F., Fernandes, F.A.N., Roque, M.C. and Rodrigues, L. 2000. Developing an educational software for heat exchangers and heat exchanger networks projects, Journal of Computer and Chemical Engineering, Vol. 24(2-7), 1247-1251.

[6] Tan, F.L. and Fok, S.C. 2006. An educational computeraided tool for heat exchanger design, Journal of Computer Application in Engineering education, Vol. 14(2), 77-89.

[7] Chapra S.C., Canale R.P. (2002) Numerical methods for engineers, $4^{\text {th }}$ Ed., Tata McGraw-Hill, New Delhi,
[8] Oko, C.O.C. (2008) Engineering computational method: An algorithmic approach, $1^{\text {st }}$ Edition, University of Port Harcourt Press, Port Harcourt.

[9] Mustafa, G. 2000. Correlations for some Thermophysical Properties of Air, International Drying Symposium, NL, Wageningen.

[10] Cengel, Y.A. (2007), Heat and mass transfer, $3^{\text {rd }}$ Ed., Tata McGram-Hill, New Delhi.

[11] Ebieto, C.E (2010) Finite element analysis of shell and tube heat exchangers, M.Eng Thesis, Department of Mechanical Engineering, University of Port Harcourt, Port Harcourt.

[12] Fakheri, A. (2003) Alternative approach for determining $\log$ mean temperature difference correction factor and number of shells of shell and tube heat exchangers, Journal of Enhanced Heat Transfer, Vol.10(4), pp. 407 420.

[13] Leong, K.C., Toh, K.C. and Leong, Y.C. 1998. Shell and tube heat exchanger design software for education application, Int. Journal of Engineering Education, Vol. 4(3), 217-224

\section{APPENDIX}

\section{Heat Exchanger Configurations}

$\boldsymbol{k}$ Heat Exchanger Configuration

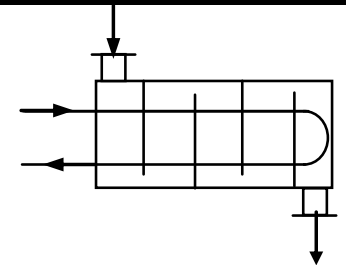

1 shell pass, 2 or even number of tube passes $(1-2 n)$

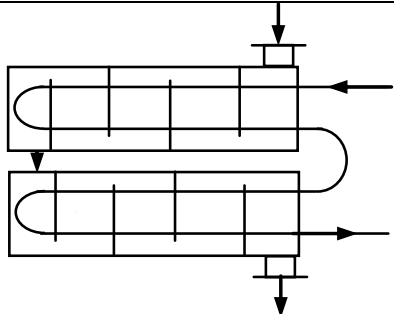

2 shell passes, 4 or multiple of 4 tube passes $(2-4 n)$

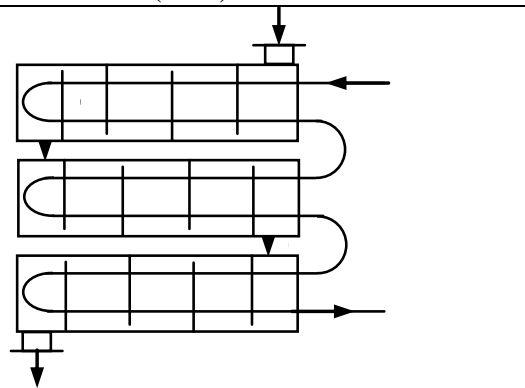

3 shell passes 6 or multiple of 6 tube passes

(3-6n) 


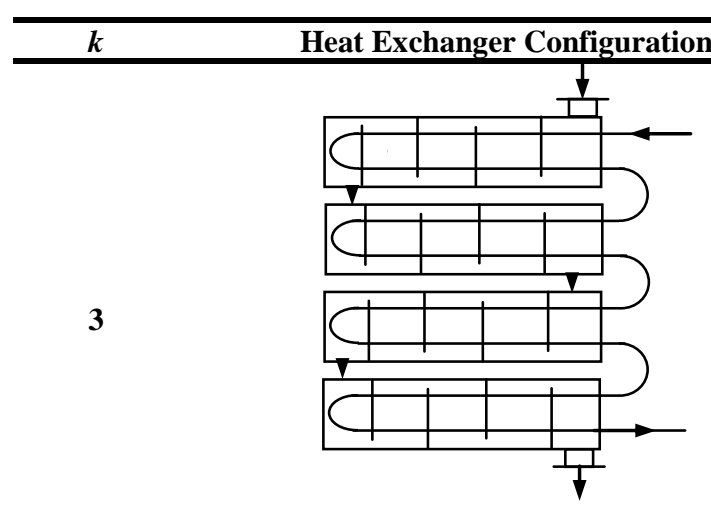

4 shell passes 8 or multiple of 8 tube passes (4-8n)

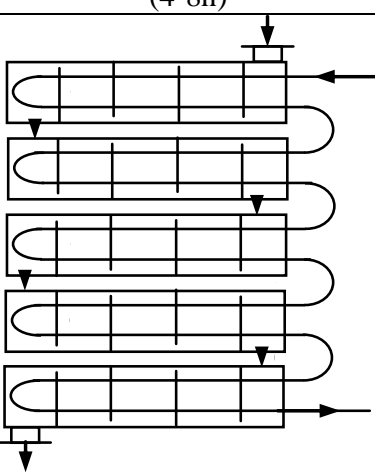

5 shell passes 10 or multiple of 10 tube passes (5-10n)

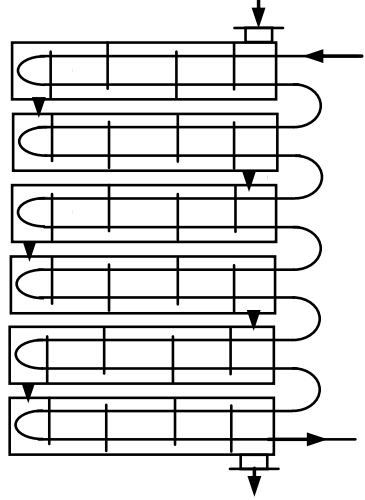

6 shell passes 12 or multiple of 12 tube passes

6

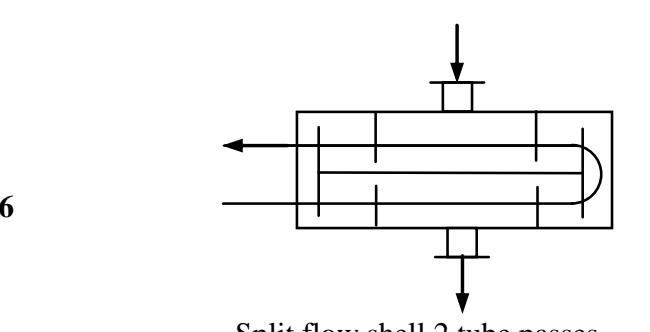

Split flow shell 2 tube passes

5

$$
\text { (6-12n) }
$$

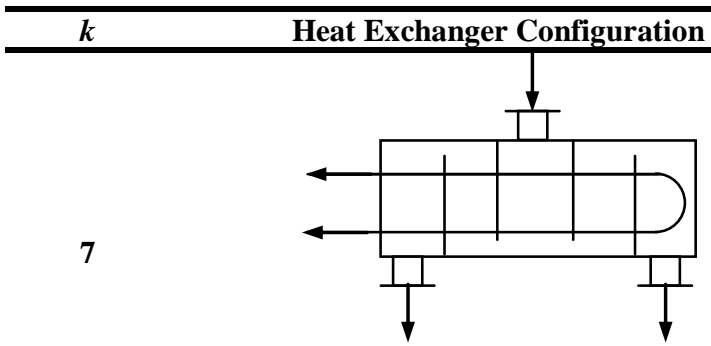

1 Divided flow shell pass even number of tube passes

8

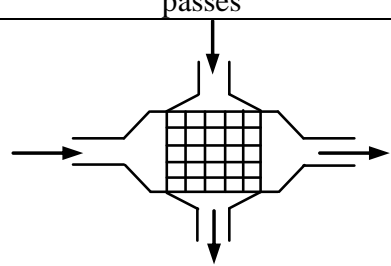

Single pass cross flow both fluid unmixed

9

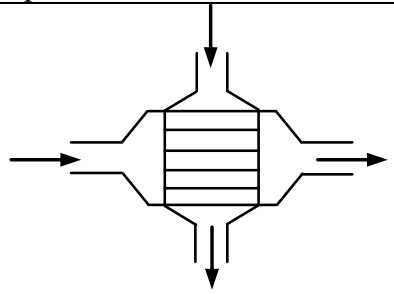

Single pass cross flow with one fluid mixed and the other unmixed

Split flow shell 2 tube passes 